\title{
Helicobacter pylori treatment with alternative drugs: good and cheap for developing countries furazolidone-based treatment- omeprazole, furazolidone and tetracycline open-label trial in a developing country: why not?
}

\begin{abstract}
Objective: To analyze eradication rate and evaluate collateral effects of an existing alternative dosage, comprised of Omeprazole, tetracycline and furazolidone for 7 days in patients with gastric and/or duodenal ulcers and first-degree relatives of family members with a diagnosis of gastric cancer with positive study for Helicobacter pylori (H. pylori) and treatment-naive.

Design and location: The out patients from the Clinic of the Gastroenterology Department of the ABC Medical School, Santo Andre, SP-Brazil.

Patients and methods: Study including treatment-naive patients with a duodenal or gastric ulcer or first-degree relatives of family members with a diagnosis of gastric cancer with a positive study for H. pylori confirmed by non-invasive: breath test (C 13), or serology (IgG) or invasive: Rapid Urease Test or gastric histology by biopsy obtained of upper gastro duodenal endoscopy (UGE). The medication schedule: Omeprazole 20mg TID (before meals) plus Tetracycline 500mg TID (after meals) and Furazolidone 200mg TID (after meals) for 7 days. Diagnostic tests for eradication rates were performed 90 days after the treatment. Adverse effects were considered as: light, mild or severe, and include: nausea, vomiting, rush, headache.
\end{abstract}

Results: 85 patients were included in the study of which 55 completed the treatment. $95 \%$ of the patients had an adequate adherence to the treatment. $49 \%$ of the patients analyzed by protocol had at least one side effect, with the most common being nausea $(24 \%)$ and there was no statistical difference between the relation of side effects and lapse of treatment for $\mathrm{H}$. pylori $5 \%$ (3 patients) interrupted the treatment. The eradication rate per protocol (PP) was $96 \%$ (95\% CI: $84 \%$ to $100 \%$ ) for patients who completed the treatment.

Conclusion: The therapy based on Omeprazole, tetracycline and furazolidone in the dosages proposed represents a good and low-cost alternative to the first-line therapy to eradicate infection by $\mathrm{H}$. pylori in treatment-naive patients in a developing country.

Keywords: helicobacter pylori, treatment, eradication, furazolidone, tetracycline, omeprazole
Volume I Issue 2 - 2014

Ana Carolina Chagas Rizzuto,' Paula Lage Pasqualucci,' Ethel Zimberg Chehter ${ }^{2}$ 'Department of Clinical Medicine, ABC Medical School, Brazil ${ }^{2}$ Department of Gastroenterology,ABC Medical School, Brazil

Correspondence: Ethel Zimberg Chehter, Department of Gastroenterology, ABC Medical School, Santo Andre, Sao Paulo, 09060-650, Brazil, Tel 5.5 II5E+1।, Email ethel.chehter@fmabc.br

Received: July 23, 2014 | Published: July 31, 2014
Abbreviations: MALT, mucosa-associated lymphoma tissue; PPI, proton pomp inhibitor; RUT, rapid urease test; UGE, upper gastro duodenal endoscopy; NSAIDs, von-steroid anti-inflammatory drugs; ITT, intention to treat; PP, per protocol; IARC, international agency for research on cancer

\section{Introduction}

Helicobacter pylori (H. pylori) is the microorganism responsible for the most frequent and persistent infection of the gastrointestinal tract worldwide. The H. pylori infection affects almost half of the world's population. In developed countries (except Japan), the prevalence is below $40 \%$, whereas in developing countries the prevalence infection is as high $90 \% .{ }^{1}$ In Brazil the prevalence of $H$. pylori infection is about $82 \%$ and mostly infected younger ages. ${ }^{2}$ The infection by Helicobacter pylori is associated to diseases like the duodenal/ gastric ulcer, acute and chronic gastritis, gastric adenocarcinoma, and mucosa-associated lymphoma (MALT) and possible B-cell gastric lymphoma and the importance of its eradication is well established [3]. According to the consensus of Maastricht III, the eradication of $H$. pylori is strongly recommended in:

a. Past or present duodenal and/or gastric ulcer, with or without complications.

b. Following resection of gastric cancer.

c. Gastric mucosa-associated lymphoma tissue (MALT) lymphoma.

d. Atrophic gastritis.

e. Functional dyspepsia.

f. Patients with first-degree relatives with gastric cancer.

g. Patient's wishes. ${ }^{4}$ 
Maastrich IV consensus suggests that the treatment regimen should be selected according to areas of low or high clarithromycin (Clari) resistance. Low prevalence of clarithromycin is defined if it is below $20 \%$ and High prevalence of clarithromycin resistance if it is higher than $20 \%$. The first-line treatment regimen in regions of low clarithromycin resistance is: Proton Pomp Inhibitor (PPI)+Clari+ Amoxicillin/Metronidazole or Bismuth Quadruple. In regions with high clarithromycin resistance the first choice is Bismuth Quadruple and if it is not available the sequential or concomitant regimens. ${ }^{5}$

What is the ideal H. pylori eradication regimen? First of all, the following factors need to be taken account when choosing a particular treatment approach: they may vary in different continents, countries, and regions:

a. Prevalence of H. pylori.

b. Prevalence of gastric cancer.

c. Resistance to antibiotics.

d. Cost level and available budget.

e. Availability of bismuth

f. Availability of endoscopy, H. pylori tests.

g. Ethnicity.

h. Drug allergies and tolerance.

i. Previous treatments outcome

j. Effectiveness of local treatment.

k. Ease of administration.

1. Adverse effects.

m. Recommended dosages and treatment duration. ${ }^{2}$

Although H. pylori is susceptible to several antimicrobials, this infection has proven challenging to cure because of increasing prevalence of bacterial strains that are resistant to the most commonly used antimicrobials, particularly to clarithromycin. An effective (i.e $>90 \%$ ) first-line therapy is mandatory for preventing the development of secondary resistance. ${ }^{6}$

Unfortunately the eradication rates following the standard triple therapy (proton pump inhibitor plus amoxicillin and clarithromycin) for $H$. pylori are declining worldwide. Several first-line strategies have been proposed to increase the eradication rate including extending the treatment duration to 14 days. ${ }^{6}$ The treatment options also suggest the use of a four drug regimen (bismuth-containing quadruple, sequential and concomitant treatments) and the use of novel antibiotics, such as fluoroquinolones. However, the efficacy of these regimens is controversial. A first-line eradication regimen should be based on what works best in a defined geographical area and must take into account the prevalence of antimicrobial resistance in that region. ${ }^{6}$

In an urban area of Brazil a recent study of the H. pylori resistance of antibiotics in adults, showed that resistance to metronidazole was $51 \%$, to clarithromycin $8 \%$, to levofloxacin $23 \%$ and to bismuth $5 \%$. There was no observed resistance to amoxicillin, tetracycline or Furazolidone. ${ }^{7}$ In 2013 another Brazilian study was done to search for the $\mathrm{H}$.pylori antibiotic resistance in children and adolescents. The mean age was $11.1 \pm 3.9$ years (3-20 years) and included 77 patients. Global rate resistance was $49.3 \%$ : $40 \%$ of strains were resistant to metronidazole, $19.5 \%$ to clarithromycin and $10.4 \%$ to amoxicillin. All of tested H. pylori strains were susceptible to furazolidone and tetracycline. They conclude that the high resistance rate to metronidazole and clarithromycin could exclude them to empirical eradication treatment and otherwise there is no resistance to furazolidone and tetracycline, so we need to think about risks and benefits of using these drugs. ${ }^{8}$

Furazolidone, a drug of the Nitrofuran class composed of a furan ring with a group of nitro, has been used in the treatment of $H$. pylori in developing countries. This drug present low bacterial resistance and it is effective against the strains of $H$. pylori and have a reduced cost. ${ }^{2,7-9}$ In 2002, Eisig et al. ${ }^{7}$ proposed a 7-day treatment based on furazolidone and tetracycline combined to a pump inhibitor (Omeprazole) in patients that had been previously treated. Not so good rates of eradication were observed $(75 \%$ by protocol and $69 \%$ with intention-to-treat), with many side effects which impeded the continued use of these medications. ${ }^{10}$

In developing countries the furazolidone is available and it is allowed to the current use. The therapeutic scheme of Omeprazole, furazolidone and tetracycline was advantageous, because it did not induce bacterial resistance, had a good adherence due to its easy posology and cost less. Nonetheless, the high rates of side effects in other studies make it necessary to search for a better-tolerated alternative. Is it a good first choice for naïve treated patients? The objective of this study was to evaluate the eradication rate and side effects of a 7-days therapeutic regimen comprising Omeprazole, tetracycline and furazolidone in a dosage alternative to the existing one in treatment-naïve patients with gastric and/or duodenal ulcers or first-degree relatives of family members with a diagnosis of gastric cancer with a positive infection for $H$.pylori.

\section{Patients and methods}

This study was done in the out-patients of the clinic of the Gastroenterology Department of the ABC Medical School (Faculdade de Medicina do ABC) between the years 2004-2011. This was an open-label and prospective trial. The study was open to those older than 18 years of age and having a duodenal or gastric ulcer or both and first-degree relatives of family members with a diagnosis of gastric cancer and positive test for $H$. pylori infection (one or two diagnostic methods positive). All patients that met the inclusion criteria and signed the consent agreement terms were included. Patients having less than 18 years, without an ulcer, testing negative for $H$. pylori, having an allergy to one of the medical components being used and those who had previously been treated for $H$. pylori with a different therapeutic scheme were excluded. The study was approved by the Research Ethics Committee of Faculdade de Medicina do ABC under number $027 / 2004$ and all the patients must signed terms of consent agreeing to participate in the study.

\section{Infectious diagnosis}

The diagnosis for $H$. pylori was realized using non- invasive tests: Urea $13 \mathrm{C}$ - breath test or antibodies for H. pylori (IgG) or invasive tests: Rapid Urease Test (RUT) or by gastric histology obtained by biopsy using an Upper Gastro duodenal Endoscopy (UGE).

\section{Treatment}

The schedule treatment consisted of 20mg Omeprazole 1 pill before each meal (breakfast, lunch and dinner), $500 \mathrm{mg}$ tetracycline-1 pill and also $200 \mathrm{mg}$ furazolidone-1 pill of each, after meals (breakfast, lunch and dinner) for 7 days (9 pills/ day). The cost of the entire treatment was approximately USD 16,00 (R $\$ 40,00$ reais, Brazilian 
money). One week later we checked: the compliance, pills count, and the correct use of medication and the occurrence of side effects. These were evaluated qualitatively as light; mild or severe (need treatment interruption/hospital care). They were: nausea, vomiting, headaches, diarrhea and others.

\section{Treatment evaluation}

The infection of H. pylori was considered cured if one of the diagnostic tests were negative (the Urea 13C- Breath Test or the Rapid Urease Test or gastric histology). The search was done 90 days after the end of treatment. The statistical determination was done using the SPSS program, software package 10.0 (SPSS Inc. USA).

\section{Results and discussion}

The sample consists of 85 patients with $H$. pylori positive in at least one diagnostic test, naive-treatment for $H$. pylori. We search for: age, gender, tobacco users (present or past, any amount of cigarette), alcohol users (present or past: more than $30-40 \mathrm{~g}$ of alcohol/more than 5 years), Regular users of non-steroid anti-inflammatory drugs (NSAIDs) and tests for $H$. pylori. In all of the patients at least one method of detecting $H$. pylori was realized and $82 \%$ (70 patients) had done two. Those that did two tests $(91 \%)$ received a positive result in both, and in $10 \%$ there was a discrepancy between the two (gastric histology was the gold-standard). Smokers (39\%) and alcohol users $(18 \%)$ were a minority, as were chronic users of NSAIDs $(26 \%)$. The clinical data of the studied population is showed in Table 1.

Table I Clinical information of the initial sample

\begin{tabular}{lll}
\hline Patients & & $\mathbf{n = 8 5}$ \\
\hline Age (Years) & & $52.8(18-84)$ \\
\hline Mean/Interval & & \\
\hline Female & $62 / 85$ & $53 \%$ \\
& & \\
Male & $23 / 85$ & $47 \%$ \\
Tobacco Users & $46 / 85$ & $39 \%$ \\
Alcohol Users & $21 / 85$ & $18 \%$ \\
NSAIDs Users & $30 / 85$ & $26 \%$ \\
\hline Initial Upper Endoscopy & & \\
\hline Duodenal Ulcer & $51 / 85$ & $60 \%$ \\
Gastric Ulcer & $20 / 85$ & $24 \%$ \\
Duodenal + Gastric Ulcer & $8 / 85$ & $9 \%$ \\
Gastritis/Esophagitis & $6 / 85$ & $7 \%$ \\
\hline
\end{tabular}

The initial upper endoscopy of the total sample is showed in Table 1: Duodenal Ulcer (DU) in 51/85 (60\%), Gastric Ulcer (GU) in $20 / 85(24 \%), \mathrm{DU} / \mathrm{GU}$ in $8 / 85(9 \%)$ and esophagitis /gastritis in $6 / 85(7 \%)$. Of the 85 patients in the sample, $55(65 \%)$ ended the treatment, took medication and underwent the final diagnostic test to assess $H$. pylori eradication. The dropout rate was $35 \%$ (30 patients). Of those that did not complete the treatment, 21 (70\%) did not return to consult number 1 (For the orientation on taking the medication) and the remainder ( 9 patients) received the orientation but got lost in the follow-up. Table 2 indicates the clinical information about the patients with complete treatment and follow-up. The initial group $(85$ patients) was similar to the final group (55 patients) including age and gender. Analyzing the final group we find $49 \%$ of DU, $31 \%$ of GU, $13 \%$ of both and other alterations (gastritis or esophagitis) $7 \%$. Initial search for $H$. pylori infection was done in at least 1 test. Forty-eight of the 55 realized 2 tests to verify the $H$. pylori infection. Of these, $88 \%$ (42 patients) had a positive result for $\mathrm{H}$. pylori in the 2 tests. Users of tobacco, alcohol and NSAIs continued being the minority (Table 2).

Table 2 Main information of final sample

\begin{tabular}{lll}
\hline Patients & \multicolumn{1}{c}{$\mathbf{n = 5 5}$} \\
\hline Age (Years) & & $53.7(18-84)$ \\
\hline Mean/Interval & & \\
\hline Female & $30 / 55$ & $55 \%$ \\
Male & $25 / 55$ & $45 \%$ \\
Tobacco Users & $18 / 55$ & $18 \%$ \\
Alcohol Users & $8 / 55$ & $15 \%$ \\
NSAIDs Users & $14 / 55$ & $25 \%$ \\
\hline Initial Upper Endoscopy & & \\
\hline Duodenal Ulcer & $27 / 55$ & $49 \%$ \\
Gastric Ulcer & $17 / 55$ & $31 \%$ \\
Duodenal + Gastric Ulcer & $7 / 55$ & $13 \%$ \\
Gastritis/Esophagitis & $4 / 55$ & $7 \%$ \\
\hline
\end{tabular}

Adequate adherence to the treatment was realized by $95 \%$ of the patients, who claimed they had taken the medication correctly, respecting the hours and doses as oriented. Three patients, 5\% (3/55), discontinued the medication or did not conclude the 7 days of treatment. None of them needed medical assistance for the collateral effects. There is no statistical evidence between the patients who took the medication correctly and had side effects and those who did not take the medication correctly and had side effects $(\mathrm{P}=0.6$ in Fisher exact test). Of the final group of 55 patients analyzed, $26(49 \%)$ had at least one side effect, with the most common being nausea which was reported by 13 patients $(24 \%)$. There was no statistical difference between the side effects and the relapse of treatment for $H$. pylori ( $\mathrm{p}=1.00$ in Fisher exact test).

Table 3 shows the distribution according to side effects presented. Other side effects reported included vertigo, sleepiness weakness, fainting, mild cramps, lower abdominal pain, dryness of the mouth, metallic taste in the mouth and itching. Of the three patients that discontinued the medication, one had nausea and vomiting, one had vertigo and one did not have any side effect. After 90 days, we did diagnostic tests to verify eradication rates of the H. pylori (Urea 13C-breath test, or Rapid Urease Test or gastric histology). The majority $(91 \%)$ had the gastric histology obtained by UGE. The eradication rate by Intention to Treat (ITT) was $62 \%(53 / 85)(95 \% \mathrm{CI}$ 0.5 - 0.73). The eradication rate Per Protocol (PP) was 96\% (53/55) (95\% CI 0.91-1.0). The high drop-out tax explained the high difference between the ITT and PP.

Table 3 Side effects of treatment omeprazole/ furazolidone/ tetracycline

\begin{tabular}{lll}
\hline Side Effects & $\mathbf{n}$ & $\%$ \\
\hline Headache & 5 & 9 \\
Nausea & 13 & 24 \\
Vomiting & 7 & 13 \\
Diarrhea & 1 & 2 \\
Others & 19 & 35 \\
At least one Side Effect & 26 & 49 \\
\hline
\end{tabular}

Table 4 shows the distribution of patients according to eradication rate. Our results showed a good rate for the eradication $H$. pylori. Besides the collateral effects, the patients could conclude the 
treatment and took correctly the medications. Is this schedule a good choice for $H$. pylori eradication? The treatment for infection by $H$. pylori has been difficult to deal because there is a growing resistance to the established antibiotics being used in numerous countries. It is therefore necessary to seek out alternative medication regimes. In the last few years, it has been observed a decrease in the eradication rates in triple therapies. The first-line therapy established world-wide to treat infection by $H$. pylori, comprised of clarithromycin, amoxicillin and metronidazole, and has declined from eradication rates of $90 \%$ down to $70-80 \%$ success rates. So, new effective therapies are needed. ${ }^{11}$

Table 4 Patients according to eradication rate

$\begin{array}{lll}\text { Intention to Treat (ITT) } & 53 / 85(62 \%) & (95 \% \mathrm{Cl} 0.5-0.73) \\ \text { Per Protocol (PP) } & 53 / 55(96 \%) & (95 \% \mathrm{Cl} 0.91-1.0)\end{array}$

Therapy failure mainly depends on both primary bacterial resistance towards antibiotics and patient compliance. In developing countries, there is a high prevalence of primary and secondary resistance to metronidazole and also to levofloxacin is present, and the bismuth compounds are very difficult to achieve. To overcome these limitations, furazolidone-based treatments have been suggested in developing countries by the World Gastroenterology Organization and Latin-America guidelines. ${ }^{2,12,13}$ On the other hand, the low rate of primary $H$. pylori resistance toward furazolidone in developed countries may render appealing the use of this drug also in these geographic areas..$^{14,15}$ Furazolidone is a synthetic nitro furan with a broad spectrum of antimicrobial activities widely used in the treatment of bacterial and protozoal infections in both humans and animals However, some concerns recently arose in using furazolidone, such as a molecule harboring a potential carcinogenetic effect. The use of Furazolidone in $H$. pylori therapies results in lots of controversy in the literature. Zullo et al. ${ }^{16}$ argued forward the potential harmful effects of the furazolidone based on studies of the literature. ${ }^{17-21}$

On the other hand Graham et al. ${ }^{14}$ questioned the warning about the carcinogenic potential of furazolidone. ${ }^{22}$ They are cautioned that furazolidone may be a carcinogen. They believed that this warning was not completely accurate citing determinations of The International Agency for Research on Cancer (IARC). It categorizes agents in categories or groups: Group 1: Carcinogenic to humans. Group 2A: Probably carcinogenic to humans (substances for which there is a lesser degree of evidence in humans but sufficient evidence in animal studies, or degrees of evidence considered appropriate to this category, e.g., unequivocal evidence of mutagenicity in mammalian cells), Group 2B: Possibly carcinogenic to humans, (substances for which there is sufficient evidence in animal tests, or degrees of evidence considered appropriate to this category), Group 3: Unclassifiable as to carcinogenicity in humans, Group 4: Probably not carcinogenic to humans. Furazolidone is a category 3 agent. The IARC report states: "Furazolidone has been produced commercially since 1955 It is used in human and veterinary medicine as an antibacterial and antiprotozoal agent. No data were available to assess the teratogenicity or chromosomal effects of this compound in humans. No case report or epidemiological study of the carcinogenicity of furazolidone was available to the Working Group. Evaluation: No evaluation of the carcinogenicity of furazolidone to experimental animals could be made. In the absence of epidemiological data, no evaluation of the carcinogenicity of furazolidone to humans could be made". ${ }^{23}$

Furazolidone was last updated in 1998 and group 3 drugs have been typically excluded from more recent complications of carcinogens.
Drugs used in animals can easily enter the human food chain and are thus rigorously controlled. As they note, furazolidone was removed from animal use. Clinical usage of all drugs is dictated by risks and benefits. Clinical usage of most drugs is often short such that the benefits can easily outweigh possible but low risks. They also questioned the situation of the metronidazole, which is listed as a definite carcinogen in animals and as a category agent $2 \mathrm{~B}$ in human and also the tinidazole: why not be worried about these drugs? Most regimens or "recipes" have never been optimized and furazolidone-containing regimens are no exception. ${ }^{24-26}$ They recommend Furazolidone combination therapy for areas with high rates of resistance and for patients with multiple prior treatment failures.

A few caveats are necessary when using furazolidone. The drug is an monoamine oxidase inhibitor and thus interacts with many other drugs and foods (e.g. we provide patients with an information sheet that cautions against aged cheese, sausage including bologna, salami and pepperoni, lima beans, lentils, snow peas, and soybeans, canned figs and raisins, beer, ale and wines, licorice, soy sauce and any food product that is made with soy sauce, monoamine oxidase inhibitors, phenylpropanolamine, ephedrine, and phenylephrine). ${ }^{22}$ They conclude that "Furazolidone is an underutilized drug especially useful in areas where resistance is widespread and multiple. To make excellent French bread requires a proven recipe; the ideal use of any anti-H .pylori therapy requires an optimized regimen. The lack of specifics by authors of the review limits its applicability for the clinician and their misunderstanding of the data about carcinogenicity unfairly maligns what should otherwise be a useful drug". ${ }^{22}$

Recently, Jiang et al. find a novel application to furazolidone: as an anti-leukemic drug in acute myeloid leukemia. In vitro studies demonstrated that furazolidone was shown to inhibit bone marrow transformation mediated by several leukemia fusion proteins and is promising drug in acute myeloid leukemia. ${ }^{27}$ In Brazil other studies were done with the same antibiotics. Our study presented a side effects rate of $49 \%$, compatible with the scientific literature, which advocates between 34-59.7\%. ${ }^{9,10}$ In Silva et al. ${ }^{10}$ the rate of reported side effects for the same therapeutic scheme was $34 \% 10$, while in Machado et al. ${ }^{9}$ it was $47.2 \%$.

In spite of the apparently high rates of side effects reported in our study, the majority corresponded to vague complaints, which might not necessarily be related to the treatment for $H$. pylori. The occurrence of side effects, nonetheless, was not the determinant factor for discontinuing the treatment, and able to consider that the proposed therapeutic scheme was well-tolerated..$^{910}$ The therapy proposed for this study avoids using clarithromycin, due to high antibiotic resistance and advocates the use of furazolidone, a drug which has proven to be efficient in fighting infection by $H$. pylori in Brazil. The low complexity of the therapeutic scheme favors patients adherence to the treatment and, consequently, success which can be seen in the high eradication rates obtained $(96 \%)$. Furthermore, this therapeutic scheme had its efficacy proved by two other studies: Silva et al. found an intention-to-treat efficacy of $69 \%$ and in Machado et al. ${ }^{9}$ H. pylori eradication rates were $83.3 \%$ patients in intention-to-treat analysis and $89.7 \%$ in per-protocol analysis. ${ }^{9,10}$

\section{Conclusion}

Most of the studies for the eradication of $H$. pylori are, as in the present study, non-comparative tests, of a single treatment and with a low population sample, which limits its generalization. Furazolidone used in patients naive to treatment has the main advantage of not 
inducing bacterial resistance, allowing for a gamut of therapeutic options if there is a need to change medication course. For these reasons, the triple medication scheme, proposed in this study, composed of omeprazole, tetracycline and furazolidone should be considered as a viable alternative for the treatment of infection by $H$. pylori in Brazil. In Brazil the furazolidone is still available, with some recommendations in national and international guidelines "the triple therapy composed of IBP, tetracycline and furazolidone as the lowcost alternative triple treatment for Brazil", we think that this is a good option: cheap, ease and effective, but we must be careful with collateral effects.

\section{Conflicts of Interest}

The author declares that there is no conflicts of interest.

\section{Acknowledgements}

None.

\section{Funding}

None.

\section{References}

1. Garza-Gonzalez E, Perz-Perz GI, Maldonado-Garza HJ, et al. A review of Helicobacter pylori diagnosis, treatment, and methods to detect eradication. World J Gastroenterol. 2014;20(6):1438-1449.

2. World Gastroenterology Organisation. WHO WGO article World Gastroenterology Organization Global Guideline: Helicobacter pylori in developing countries. J Clin Gastroenterol. 2011;45(5):383-388.

3. Wu W, Yang Y, Sun G. Recent insights into antibiotic resistance in Helicobacter pylori eradication. Gastroenterol Res Pract. 2012: 723183 .

4. Malfertheiner P, Megraud F, O'Morain C, et al. Current concepts in the management of Helicobacter pylori infection: the Maastricht III Consensus Report. Gut. 2007;56(6):772-781.

5. Malfertheiner P, Megraud F, O'Morain CA, et al. Management of Helicobacter pylori infection-the Maastricht IV/ Florence Consensus Report. Gut. 2012;61(5):646-664.

6. Frederico A, Gravina AG, Miranda A, et al. Eradication of Helicobacter pylori infection: which regimen first? World $J$ Gastroenterol. 2014;20(3):665-672.

7. Eisig JN, Silva FM, Barbuti RC, et al. Helicobacter pylori antibiotic resistance in Brazil: clarithromycin is still a good option. Arq Gastroenterol. 2011;48(4):261-264.

8. Ogata SK, Godoy AP, da Silva Patricio FR, et al. High Helicobacter pylori resistance to metronidazole and clarithromycin in Brazilian children and adolescents. J Pediatr Gastroenterol Nutr. 2013;56(6):645648.

9. Machado RS, da Silva MR, Viriato A. Furazolidone, tetracycline and omeprazole: a low-cost alternative for Helicobacter pylori eradication in children. J Pediatr (Rio J). 2008;84(2):160-165.

10. Silva FM, Eisig JN, Chehter EZ, et al. Omeprazole, Furazolidone and Tetracycline: an eradication treatment for resistant H. pylori in Brazilian patients with peptic ulcer disease. Rev Hosp Clin Fac Med Sao Paulo. 2002;57(5):205-208.
11. Buzzas GM. First-line eradication of Helicobacter pylori: are the standard triple therapies obsolete? A different perspective. World $J$ Gastroenterol. 2010;16(31):3865-3870.

12. Coelho LG, Maguilnik I, Zaterka S, et al. 3rd Brazilian consensus on Helicobacter pylori. Arq Gastroenterol. 2013;50(2).

13. Coelho LG, Leon-Barua R, Quigley EM. Latin-American Consensus Conference on Helicobacter pylori infection: Latin-American National Gastroenterological Societies affiliated with the Inter-American Association of Gastroenterology (AIGE). Am $J$ Gastroenterol. 2000;95(10):2688-2691.

14. Graham DY, Osato MS, Hoffman J, et al. Furazolidone combination therapies for Helicobacter pylori infection in the United States. Aliment Pharmacol Ther. 2000;14(2):211-215.

15. Van Zwet AA, Thijs JC, van der Wouden EJ, et al. Low cure rate of Helicobacter pylori infection with omeprazole and furazolidone dual therapy for one week. Aliment Pharmacol Ther. 1997;11(3):533-535.

16. Zullo A, Ierardi E, Hassan C, et al. Furazolidone-based therapies for Helicobacter pylori infection: a pooled-data analysis. Saudi J Gastroenterol. 2012;18(1):11-17.

17. Ahmed HH, El-Aziem SH, Abdel-Wahhab MA. Potential role of cysteine and methionine in the protection against hormonal imbalance and mutagenicity induced by furazolidone in female rats. Toxicology. 2008;243(1-2):31-42.

18. Ali BH. Pharmacological, therapeutic and toxicological properties of furazolidone: some recent research. Vet Res Commun. 1999; 23(6):343360 .

19. Madrigal-Bujaidar E, Ibanez JC, Cassani M, et al. Effect of furazolidone on sister-chromatid exchanges, cell proliferation kinetics, and mitotic index in vivo and in vitro. J Toxicol Environ Health. 1997;51(1):89-96.

20. Jin X, Tang S, Chen Q, et al. Furazolidone induced oxidative DNA damage via up-regulating ROS that caused cell cycle arrest in human hepatoma G2 cells. Toxicol Lett. 2011;201(3):205-212.

21. Vincentini O, De Angelis I, Stammati A, et al. Functional alterations induced by the food contaminant furazolidone on the human tumoral intestinal cell line Caco-2. Toxicol in vitro. 1993;7(4):403-406.

22. Graham D, Lu H. Furazolidone in Helicobacter pylori therapy: misunderstood and often unfairly maligned drug told in a story of French bread. Saudi J Gastroenterol. 2012;18(1):1-2.

23. WHO. Some Food additives, feed additives and naturally occurring substances. IARC monographs on the evaluation of carcinogenic risks to humans, WHO, Geneva. 1983; p. 141.

24. Graham DY. Efficient identification and evaluation of effective Helicobacter pylori therapies. Clin Gastroenterol Hepatol. 2009; 7(2):145-148.

25. Graham DY, Fischbach L. Helicobacter pylori treatment in the era of increasing antibiotic resistance. Gut. 2010;59(8):1143-1153.

26. Rimbara E, Fischbach LA, Graham DY. Optimal therapy for Helicobacter pylori infections. Nat Rev Gastroenterol Hepatol. 2011;8(2):79-88.

27. Jiang X, Sun L, Qiu JJ, et al. A novel application of furazolidone: anti-leukemic activity in acute myeloid leukemia. PLoS One. 2013;8(8):e72335. 